\title{
Clinical Characteristics and Risk Factors for Pneumocystis Jirovecii Pneumonia during Immunosuppressive Treatment in Patients with Ulcerative Colitis: A Retrospective Study
}

\author{
Kentaro Kojima ${ }^{1,2}$, Toshiyuki Sato ${ }^{3}$, Motoi Uchino ${ }^{4}$, Yoko Yokoyama ${ }^{1}$, Tetsuya Takagawa ${ }^{1}$, Yoshio Ohda ${ }^{5}$, Nobuyuki \\ Hida $^{1}$, Kenji Watanabe ${ }^{3}$, Kazutoshi Hori ${ }^{1}$, Hiroto Miwa ${ }^{5}$, Hiroki Ikeuchi ${ }^{4}$, Shiro Nakamura ${ }^{1}$, Masahito Shimizu $^{2}$
}

\author{
1) Division of Internal \\ Medicine, Department of \\ Inflammatory Bowel Disease, \\ Hyogo College of Medicine, \\ Hyogo \\ 2) Department of \\ Gastroenterology and Internal \\ Medicine, Gifu University \\ Graduate School of Medicine, \\ Gifu \\ 3) Department of Intestinal \\ inflammation Research, Hyogo \\ College of Medicine, Hyogo \\ 4) Division of Surgery, \\ Department of Inflammatory \\ Bowel Disease, Hyogo College \\ of Medicine, Hyogo \\ 5) Division of \\ Gastroenterology, Department \\ of Internal Medicine, Hyogo \\ College of Medicine, Hyogo, \\ Japan
}

\section{Address for correspondence: Toshiyuki Sato \\ Department of Intestinal Inflammation Research, Hyogo College of Medicine, Mukogawa-cho Nishinomiya- shi, Hyogo, 663-8501, Japan tshnngn@hyo-med.ac.jp}

Received: 18.02.2020 Accepted: 18.05.2020

\section{ABSTRACT}

Aims: This study aimed to clarify the clinical characteristics of Pneumocystis jirovecii pneumonia (PJP) infection in patients with ulcerative colitis (UC) and to identify risk factors for PJP using a retrospective case-control study.

Methods: Of 4,525 patients with UC treated between 2007 and 2019, we identified those who satisfied the criteria for PJP. The Lichtiger clinical activity index (LCI) was compared between the initiation of immunosuppressive drug treatment and the onset of PJP. A retrospective case-control study was conducted using a PJP group and a non-PJP group.

Results: Nine patients experienced PJP, of whom two died. Since October 2014, there were no cases of PJP among UC patients aged $\geq 50$ years who were prescribed three or more immunosuppressive agents given prophylactic sulfamethoxazole-trimethoprim (TPM-SMX). The median LCI (range) was 13 (8-17) at the initiation of treatment versus $2(1-8)$ at PJP onset $(\mathrm{p}=0.016)$. The median time to PJP onset was 83 days after treatment initiation. In the PJP group the median age was significantly greater $(p=0.022)$, three immunosuppressants were used significantly more frequently $(\mathrm{p}=0.004)$, and the lymphocyte counts during treatment were significantly lower $(\mathrm{p}<0.01)$ than in the non-PJP group. The cut-off lymphocyte count that distinguished PJP patients from non-PJP patients was $570 / \mu \mathrm{L}$ according to a receiver-operating curve analysis. Conclusions: Prophylactic administration of TPM-SMX prevented further cases of PJP. The onset of PJP occurred at the same time as the symptoms of UC were stabilizing and the immunosuppressive drugs were being reduced. Greater age, lower lymphocyte count, and treatment with three immunosuppressive drugs were risk factors for PJP.

Key words: ulcerative colitis - Pneumocystis jirovecii pneumonia - inflammatory bowel diseases - casecontrol study.

Abbreviations: HIV: human immunodeficiency virus; IBD: inflammatory bowel disease; LCI: Lichtiger clinical activity index; Pneumocystis jirovecii pneumonia (PJP); PSL: prednisolone; RA: rheumatoid arthritis; UC: ulcerative colitis; TMP-SMX: sulfamethoxazole-trimethoprim; TNF- $\alpha$ : tumor necrosis factor- $\alpha$.

\section{INTRODUCTION}

Pneumocystis jirovecii pneumonia (PJP) is an opportunistic infection in patients infected with human immunodeficiency virus (HIV). However, with the increasing use of various immunosuppressant drugs today, the number of patients with PJP not associated with HIV is increasing. PJP is usually classified into two types: PJP with HIV (HIV-PJP) and PJP without HIV (non-HIV-PJP), which is often associated with a collagen-based disease or malignant tumor. The two types of disease differ greatly in their pathophysiology and clinical characteristics $[1,2]$. Respiratory failure is known to progress more rapidly and to require more artificial respiratory control, resulting in a poor prognosis in non-HIV-PJP rather than in HIV-PJP [3-5].

In Japan, the main treatment strategy for ulcerative colitis (UC) before the 1990s was based on the use of prednisolone (PSL). Since then, various kinds of immunosuppressant drugs, such as thioprine, calcineurin inhibitors, and anti-tumor necrosis factor- $\alpha$ (TNF- $\alpha$ ), have come into use. Tofacitinib, which is used to treat rheumatoid arthritis (RA), has been available for UC in Japan since July 2018. Winthrop et al. 
[6] reported that of 58 patients with opportunistic infections among 5,761 tofacitinib-treated patients with RA, four were infected with PJP and one died. Therefore, there is still some concern that PJP can arise in patients with UC treated with tofacitinib. Because non-HIV-PJP arises from treatment with immunosuppressive drugs, it is essential to investigate the risk factors for PJP in patients with UC. Case-series of PJP in patients with inflammatory bowel disease (IBD) are scarce, and few controlled studies have investigated risk factors for the onset of PJP in patients with UC $[7,8]$.

The purpose of this study was to clarify the clinical characteristics of patients with PJP and to identify the risk factors for PJP in patients with UC, using a retrospective case-control study.

\section{METHODS}

This study was approved by the Institutional Ethics Committee at our institute (number 2650). We searched the records of patients $(\mathrm{n}=4,525)$ with UC who received treatment in our hospital between April 2007 and March 2019. Of these patients, 81 were diagnosed with pulmonary inflammatory diseases and we extracted the records of patients with PJP.

\section{Diagnosis of PJP}

The criteria for a diagnosis of PJP are: (1) Pneumocystis jirovecii detected by Giemsa staining or Grocott staining using sputum or DNA polymerase chain reaction of bronchoalveolar lavage (BAL) fluid; (2) serum $1,3-\beta-D$-glucan $\geq 31.1 \mathrm{pg} /$ $\mathrm{mL}$ on the Wako $\beta$-glucan test; and (3) diffuse and relatively homogeneous ground-glass opacities and/or consolidation dominated in both lungs on chest computed tomography. Definite PJP was diagnosed in patients who met all three criteria, while patients who met the last two criteria were diagnosed with probable PJP. For the second criteria we used the Wako $\beta$-glucan test because it was reported to most reliably measure serum $1,3-\beta-\mathrm{D}$-glucan; the sensitivity and specificity of the cut-off value $(31.1 \mathrm{pg} / \mathrm{mL})$ in predicting PJP are $92.3 \%$ and $86.1 \%$, respectively [9]. The severity of PJP was evaluated based on chest radiography and computed tomography findings [10] and the degree of acute respiratory distress syndrome [11].

\section{Severity of UC}

The severity of UC was assessed with the Lichtiger clinical activity index (LCI), described by Lichtiger et al. [12]. The LCI includes the frequency of diarrhea/day, the presence or absence of nocturnal diarrhea, visible blood in the stool, fecal incontinence, abdominal pain or cramping, general well-being, abdominal tenderness, and requirement for antidiarrheal medication (Table I). The LCI is calculated and converted to a category of UC severity. The maximum score is 21 and a score of $\geq 10$ is defined as severe UC. The LCI can be used to verify the progress of UC symptoms. To investigate the relationship between PJP onset and the severity of UC symptoms, the LCI score was compared in each patient: at the commencement of immunosuppressants and at the onset of PJP.

\section{Day of PJP onset after immunosuppressant therapy}

We used the Kaplan-Meier estimator to investigate the risk of PJP onset following the commencement of immunosuppressive treatment.

\section{Case-control study}

We used a case-control study to investigate the risk factors for PJP. Non-PJP patients with UC were matched to PJP patients using the following three criteria. First, we identified patients hospitalized as an inpatient or those undergoing treatment in an outpatient clinic on the same date as a patient with PJP was being treated. Second, non-PJP patients were matched with PJP patients by gender. Third, only non-PJP patients who had not been treated with prophylactic sulfamethoxazoletrimethoprim (TPM-SMX) were enrolled. Patients were not matched by age because we considered that age should be evaluated as a possible risk factor for PJP.

We planned to match non-PJP patients to PJP patients at a ratio of 5:1 in order to maximize the statistical accuracy [13]. The following factors were included in univariate analyses to compare the PJP and non-PJP groups: age, duration of $\mathrm{UC}$, disease location at the beginning of immunosuppressive therapy, albumin, hemoglobin, C-reactive protein, white blood cell count, lymphocyte count and neutrophil count at the beginning of immunosuppressive therapy, lowest lymphocyte count $(/ \mu \mathrm{L})$ during treatment; total dose of PSL, duration of PSL administration (in days), and the number of immunosuppressant drugs given.

Table I. Lichtiger clinical activity index score for ulcerative colitis [12]

\begin{tabular}{|c|c|c|c|c|c|c|}
\hline \multirow[t]{2}{*}{ Variable } & \multicolumn{6}{|c|}{ Scores } \\
\hline & 0 & 1 & 2 & 3 & 4 & 5 \\
\hline $\begin{array}{l}\text { Diarrhea (number of daily } \\
\text { stools) }\end{array}$ & $0-2$ & $3-4$ & $5-6$ & $7-9$ & $\geq 10$ & \\
\hline Nocturnal diarrhea & No & Yes & & & & \\
\hline $\begin{array}{l}\text { Visible blood in stool (\% of } \\
\text { movements) }\end{array}$ & 0 & $<50$ & $\geq 50$ & 100 & & \\
\hline Fecal incontinence & No & Yes & & & & \\
\hline Abdominal pain or cramping & None & Mild & Moderate & Severe & & \\
\hline General well-being & Perfect & Very good & Good & Average & Poor & Terrible \\
\hline Abdominal tenderness & No & $\begin{array}{l}\text { Mild and } \\
\text { localized }\end{array}$ & $\begin{array}{l}\text { Mild to moderate } \\
\text { and diffuse }\end{array}$ & $\begin{array}{l}\text { Severe or } \\
\text { rebound }\end{array}$ & & \\
\hline Need for antidiarrheal drugs & No & Yes & & & & \\
\hline
\end{tabular}


We also performed receiver-operating characteristic (ROC) curve analysis to investigate the cut-off value for the lowest lymphocyte count that would predict PJP.

\section{Statistical analysis}

We used the Mann-Whitney $U$ test to compare the continuous nonparametric variables for patients in the nonPJP and PJP groups. The Wilcoxon signed-rank test was used to compare the parametric variables between the two groups. Fisher's exact test was used to compare categorical variables. Because the number of patients in the PJP group was too small for multivariable analysis, we only conducted univariate analyses. A ROC curve was used to determine the cut-off value for the lowest lymphocyte count during treatment that would distinguish the PJP and non-PJP groups. We calculated the overall day of PJP onset using the Kaplan-Meier estimator. Statistical tests and the ROC analysis were performed with EZR ver. 1.36 [14]. For each analysis, $\mathrm{p}<0.05$ was considered statistically significant.

\section{RESULTS}

\section{Clinical characteristics of patients with PJP}

There were no cases of PJP between October 2014 to 2019, when TPM-SMX was administered prophylactically to prevent PJP in UC patients aged $\geq 50$ years prescribed more than three immunosuppressive agents (PSL, calcineurin inhibitor, antiTNF- $\alpha$, or thioprine).

All cases of PJP were identified between 2007 and 2014. Nine patients $(0.2 \%)$ were diagnosed with PJP; three patients with definite PJP: six patients with probable PJP did not receive BAL because of the poor unstable respiration status or because we were unable to obtain the patient's consent.

The clinical characteristics of the nine patients diagnosed with PJP are shown in Table II. All nine PJP patients were given PSL. The greatest number of immunosuppressive agents administered, including PSL, was three in five patients, two in two patients, and one (PSL alone) in two patients. Among the five patients using three immunosuppressives, thioprine was used by all five, calcineurin inhibitors by four, and anti-TNF- $\alpha$ by one. The severity of PJP in individual patients is shown in
Table III. The median value of $\beta$-D-glucan in these patients was $149.1 \mathrm{pg} / \mathrm{mL}$ (69.5-421.5). Of the nine patients with PJP, two died.

\section{Comparison of LCI at the initiation of treatment and} at PJP onset

LCI could not be measured at PJP onset in two of the nine patients with PJP: one patient with advanced uterine cancer had undergone total hysterectomy and construction of an artificial anus using the colon. The other patient, with severe UC, had undergone ileal pouch-anal anastomosis. Therefore, seven patients were eligible for the comparison of LCI at the initiation of immunosuppression and at PJP onset. The median LCI (range) at the initiation of treatment was 13 (8-17), and at PJP onset was $2(1-8)$, indicating a significant reduction ( $\mathrm{p}$ $=0.016)$ (Fig. 1).

\section{Onset of PJP after immunosuppressive treatment}

A Kaplan-Meier analysis of the nine patients with PJP showed that the median period from the beginning of immunosuppressive treatment to PJP onset was 83 days (Fig. 2).

\section{Case-control study}

Although we tried to match non-PJP to PJP patients at a ratio of 5:1, we could not match five non-PJP patients to one PJP patient due to the limitations imposed by the matching criteria. Therefore, we matched non-PJP patients to PJP patients at a ratio of 4:1 resulting in 36 patients in the nonPJP group. Table IV shows the comparison of data from the PJP and non-PJP groups. The median age of the patients was significantly greater in the PJP group than in the non-PJP group $(p=0.022)$. There were no significant differences between the two groups in the duration of UC and the disease location at the beginning of immunosuppressive therapy. Although the laboratory data at the beginning of immunosuppressive therapy were not significantly different between the two groups, the lowest lymphocyte count during treatment was significantly lower in the PJP group than in the non-PJP group $(\mathrm{p}<0.001)$. Fig. 3 shows the ROC analysis used to determine the cut-off value for the lowest lymphocyte count that distinguished the

Table II. Baseline characteristics of patients with Pneumocystis jirovecii pneumonia (PJP)

\begin{tabular}{|c|c|c|c|c|c|c|c|}
\hline Case & $\begin{array}{c}\text { Year of } \\
\text { PJP onset }\end{array}$ & $\begin{array}{c}\text { Prophylactic } \\
\text { administration of } \\
\text { TPM-SMX }\end{array}$ & $\begin{array}{c}\text { PSL } \\
\text { (mg/day) }\end{array}$ & Thioprine & $\mathrm{CNI}$ & anti-TNF- $\alpha$ & $\begin{array}{l}\text { Number of } \\
\text { ISDs }\end{array}$ \\
\hline 1 & 2007 & - & $60 \rightarrow 20$ & + & + & - & 3 \\
\hline 2 & 2007 & - & $80 \rightarrow 30$ & + & - & - & 2 \\
\hline 3 & 2008 & - & $60 \rightarrow 15$ & - & + & - & 2 \\
\hline 4 & 2008 & - & $40 \rightarrow 5$ & - & - & - & 1 \\
\hline 5 & 2009 & - & $50 \rightarrow 15$ & + & + & - & 3 \\
\hline 6 & 2009 & - & $60 \rightarrow 20$ & + & + & - & 3 \\
\hline 7 & 2013 & - & $60 \rightarrow 20$ & + & + & - & 3 \\
\hline 8 & 2013 & - & $40 \rightarrow 20$ & - & - & - & 1 \\
\hline 9 & 2014 & - & $20 \rightarrow 7.5$ & + & - & + & 3 \\
\hline
\end{tabular}

TPM-SMX: sulfamethoxazole/trimethoprim; PSL: prednisolone [initial (maximum) dose $\rightarrow$ dose at PJP onset]; CNI: calcineurin inhibitor; TNF: tumor necrosis factor; ISDs: immunosuppressive drugs. 
Table III. Severity of Pneumocystis jirovecii pneumonia in individual patients

\begin{tabular}{|c|c|c|c|c|c|c|c|c|c|c|}
\hline \multirow{3}{*}{ Case } & \multirow{3}{*}{$\begin{array}{l}\beta-\mathrm{d}-\mathrm{G}^{\mathrm{a}} \\
(\mathrm{pg} / \mathrm{ml})\end{array}$} & \multicolumn{4}{|c|}{ Radiological findings } & \multicolumn{4}{|c|}{ ARDS } & \multirow{3}{*}{ Outcome } \\
\hline & & \multicolumn{3}{|c|}{ GGO } & \multirow[t]{2}{*}{ Consolidation } & \multirow{2}{*}{$\begin{array}{c}\mathrm{PaO}_{2} \\
(\mathrm{mmHg})\end{array}$} & \multirow[t]{2}{*}{$\mathrm{FiO}_{2}$} & \multirow[t]{2}{*}{$\mathrm{P} / \mathrm{F}$ ratio } & \multirow[t]{2}{*}{ Severity } & \\
\hline & & Diffuse & Patchy & Extensive & & & & & & \\
\hline 1 & 185.4 & + & + & - & - & - & - & - & - & Cured \\
\hline 2 & 147.2 & + & + & - & - & - & - & - & - & Cured \\
\hline 3 & 149.1 & + & + & + & + & 49.6 & 0.9 & 55.1 & severe & Died \\
\hline 4 & 405.7 & + & + & + & + & 43.9 & 0.8 & 54.9 & severe & Died \\
\hline 5 & 112.5 & + & - & - & - & - & - & - & - & Cured \\
\hline 6 & 69.5 & + & + & + & + & 53.5 & 0.60 & 89.2 & severe & Cured \\
\hline 7 & 421.5 & + & - & + & - & 117.2 & 0.21 & 558.1 & - & Cured \\
\hline 8 & 72.8 & + & + & + & - & 54.7 & 0.90 & 60.8 & severe & Cured \\
\hline 9 & 174.2 & + & + & + & - & 74.6 & 0.21 & 355.2 & - & Cured \\
\hline
\end{tabular}

$\beta-\mathrm{D}-\mathrm{G}=1,3-\beta$-D-glucan; $\mathrm{GGO}=$ ground glass opacity; $\mathrm{PaO}_{2}$ : partial pressure of oxygen in arterial blood; $\mathrm{FiO}_{2}$ : fraction of inspiratory oxygen; $\mathrm{PF}$ ratio $=\mathrm{PaO}_{2} / \mathrm{FiO}_{2}$; ARDS: acute respiratory distress syndrome; mild ARDS: PF ratio 200 to 300; moderate ARDS: PF ratio 100 to 200; severe ARDS: PF ratio $<100$. ${ }^{a}$ Wako $\beta$-glucan test

Lichitiger clinical activity index $(\mathrm{n}=7)$

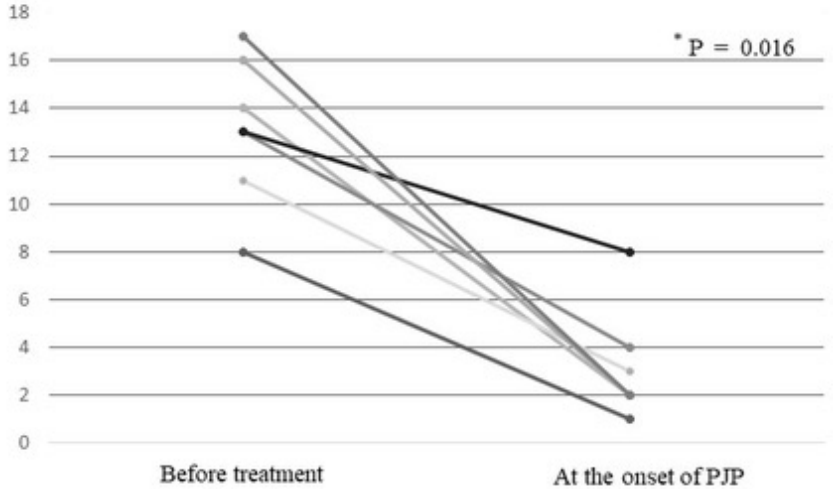

Fig. 1. Comparison of Lichtiger clinical activity index before treatment and at the onset of Pneumocystis jirovecii pneumonia (PJP). Median score (range) 13 (8-17) before treatment was significantly reduced to $2(1-8)$ at the onset of PJP.

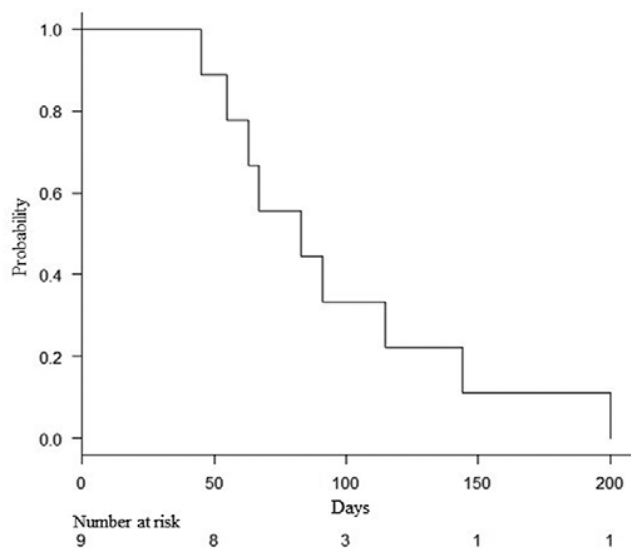

Fig. 2. Kaplan-Meier analysis of the period from the beginning of immunosuppressive treatment to the onset of Pneumocystis jirovecii pneumonia. The median was 83 days.

Table IV. Comparison of disease and treatment characteristics between patients with or without Pneumocystis jirovecii pneumonia

\begin{tabular}{|c|c|c|c|}
\hline & PJP group $(n=9)$ & Non-PJP group $(\mathrm{n}=36)$ & $\mathrm{p}$ \\
\hline Age (years) & $56(54-62)$ & $39(25-54)$ & 0.022 \\
\hline \multicolumn{4}{|l|}{ Disease location at the beginning of ISDs, $\mathrm{n}(\%)$} \\
\hline Extensive colitis & $7(77.8)$ & $21(58.3)$ & 0.447 \\
\hline Left-sided colitis & $2(22.2)$ & $15(41.7)$ & \\
\hline Duration of UC, month & $38(24-47)$ & $32.5(10.8-94.5)$ & 0.787 \\
\hline \multicolumn{4}{|l|}{ Laboratory tests at the beginning of ISDs } \\
\hline $\mathrm{Alb}(\mathrm{g} / \mathrm{dl})$ & $3.5(3.05-3.7)$ & $3.1(2.80-3.7)$ & 0.451 \\
\hline HGB (g/dl) & $11.2(10.5-11.4)$ & $11.8(9.9-13.3)$ & 0.932 \\
\hline $\mathrm{CRP}(\mathrm{mg} / \mathrm{l})$ & $1.9(0.5-4.28)$ & $1.4(0.9-2.9)$ & 0.733 \\
\hline 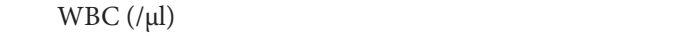 & $7780(6810-11590)$ & 7885 (7122.5-10125) & 0.777 \\
\hline $\operatorname{Lym}(/ \mu \mathrm{l})$ & $1080.7(551-1367)$ & $1138.2(791.8-1662.4)$ & 0.444 \\
\hline $\operatorname{Neu}(/ \mu \mathrm{l})$ & $5513.1(4961-6263)$ & $5972.5(4612.1-7644)$ & 0.755 \\
\hline Lowest lymphocyte count during treatment with ISDs $(/ \mu \mathrm{L})$ & $405.9(260.3-521.7)$ & $808.5(627.9-1167.1)$ & $<0.001$ \\
\hline Cumulative dose of PSL given (mg) & $2360(1980-2765)$ & $1777.5(1257.5-2647.5)$ & 0.128 \\
\hline The duration of PSL administration (days) & $59(58-77)$ & $78(55-102)$ & 0.643 \\
\hline \multicolumn{4}{|l|}{ Number of ISDs, n (\%) } \\
\hline 1 drug or 2 drugs & $4(44.4)$ & $33(91.7)$ & 0.004 \\
\hline 3 drugs & $5(55.6)$ & $3(8.3)$ & \\
\hline
\end{tabular}

Values are presented as number (\%) or median (interquartile range). UC: ulcerative colitis; ISD: immunosuppressive drugs; Alb: albumin; HGB: hemoglobin; CRP: C-reactive protein; WBC: white blood cell; lym: lymphocyte; neu: neutrophil; PSL: prednisolone 
PJP patients from the non-PJP patients during treatment. The ROC analysis confirmed the significance of the lowest lymphocyte count during treatment, with an area under the curve of 0.86 (95\% CI, 0.74-0.99). The cut-off value, sensitivity, and specificity for the lowest lymphocyte count to predict PJP during treatment were $570 / \mu \mathrm{L}, 81 \%$ and $89 \%$ respectively. There were no significant differences between the two groups in terms of the total dose of PSL or the duration of PSL administration. Three immunosuppressive drugs were used significantly more frequently than one or two drugs in the PJP group than in the non-PJP group $(\mathrm{p}=0.004)$.

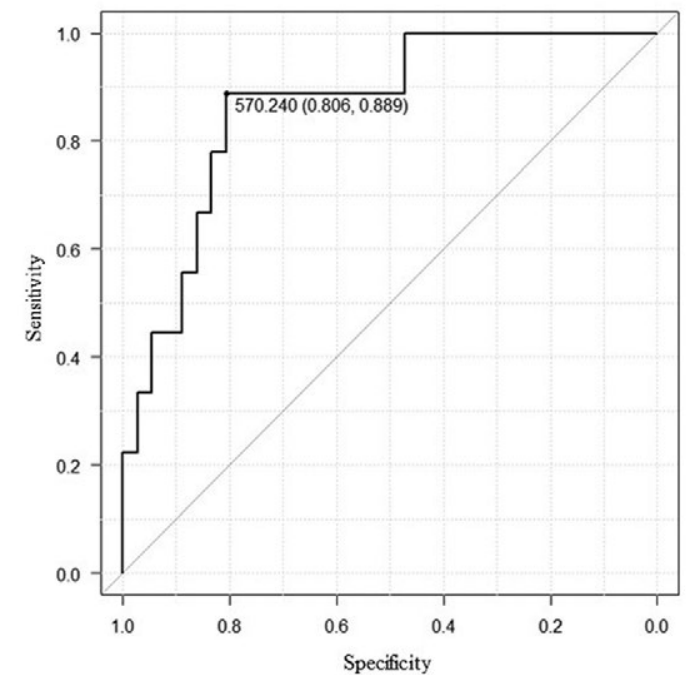

Fig. 3. Receiver-operating characteristic curve of the lowest lymphocyte count in the whole blood during treatment predicts the onset (or not) of Pneumocystis jirovecii pneumonia. Area under curve 0.864 (95\% CI: 0.738-0.991), cut-off value $570 / \mu \mathrm{L}$, sensitivity 0.806 , and specificity 0.889 .

\section{DISCUSSION}

For patients with IBD, there is no consensus on whether to start prophylactic TPM-SMX or its administration frequency. It is not recommended to administer TPM-SMX indiscriminately to all patients taking immunosuppressive therapy because resistant strains may emerge and there is a risk of side effects of TPM-SMX. Since October 2014, we have considered it necessary to administer prophylactic TPM-SMX to patients with UC aged $\geq 50$ years who are prescribed three or more immunosuppressive drugs. This decision was based on a study published in 2011 showing that prophylactic TPMSMX suppresses the onset of PJP [15]. Since implementing this treatment, there have been no further cases of PJP among patients with UC. Therefore, we believe this is an important treatment option.

By comparing the LCI at the beginning of immunosuppressive treatment and at PJP onset, this study shows that PJP onset occurred after the activity of UC was reduced. The median days from the beginning of immunosuppressive therapy to PJP onset was 83 days, which indicates that PJP onset did not occur early in the intensive treatment of UC, but relatively late in the treatment, when the UC symptoms were relieved.
The relationship between UC activity and PJP onset has not been reported previously. The mechanism underlying the alveolar damage caused by PJP originates from an excessive immune response to the fungus Pneumocystis jirovecii $[2,16]$. When the active symptoms of UC settle down, it is common to reduce the dose of immunosuppressant drugs. This implies that the reduction in the immunosuppressant drugs induces the activation of the immune response to Pneumocystis jirovecii , leading to alveolar damage. In other words, we consider that the onset of PJP occurs at the same time as the symptoms of UC are becoming stabilized and when the immunosuppressant drugs are reduced.

Based on the clinical features of PJP in patients with UC, the risk factors identified in this case-control study were advanced age, a reduced lymphocyte count, and the use of three immunosuppressants. Harigai et al. [17] reported that among patients with RA treated with immunosuppressants, those aged $\geq 65$ years had PJP significantly more frequently than those aged $<65$ years (hazard ratio $=3.77,95 \%$ confidence interval: 1.54-9.25) [17]. Long et al. [7] demonstrated that among patients with IBD, the rate of PJP was greater in patients aged $\geq 60$ years, although their outcome was not derived by comparison but derived by a case series. In the present study, the age of the PJP group was also significantly greater than that of the non-PJP group $(\mathrm{p}=0.022)$, indicating similar results to those of previous reports.

In this study, the lowest lymphocyte count during treatment was significantly lower in the PJP group than in the nonPJP group. A ROC analysis identified the cut-off value for lymphocytes that predicted PJP as $570.2 / \mu \mathrm{L}$. The lymphocyte count is reduced in patients with PJP and hematological malignancies, including patients with non-HIV-PJP [18]. Based on the data of patients with Wegener's granulomatosis, Okafor et al. [19] proposed that TPM-SMX should be given to UC patients with lymphocyte counts $<600 / \mu \mathrm{L}$. Although their proposal was not based on patients with UC, our estimated cut-off value for lymphocyte count that predicts PJP, which has not yet been reported for PJP associated with IBD, supports their proposal.

All nine patients in the PJP group were given PSL. Although no significant difference was detected between the PJP and non-PJP groups in either the total dose of PSL administered or the duration of PSL administration, the lowest lymphocyte count during treatment was significantly lower in the PJP group than in the non-PJP group. In addition, these patients developed PJP a long time after starting immunosuppressive therapy of a median of 83 days based on the Kaplan-Meier estimate (Fig. 2). These results suggest that we should pay close attention to patients who have received PSL for a long time, especially if their lymphocyte count has decreased.

Three immunosuppressive drugs rather than one or two were used significantly more frequently in the PJP group than in the non-PJP group $(\mathrm{p}=0.004)$. The guideline issued by the European Crohn's Disease and Colitis Organization, based on the data acquired from patients with leukemia, hematological malignancy, or organ transplantation, describes the use of immunosuppressive drugs and prophylactic TPM-SMX for patients with inflammatory bowel disease. It states that when two immunosuppressive drugs are used, prophylactic 
administration of TPM-SMX should be considered and, when three immunosuppressive drugs (including calcineurin inhibitor or anti-TNF- $\alpha$ ) are used, prophylactic administration of TPM-SMX is recommended [20]. Our results support this recommendation in patients with UC. However, two patients in the PJP group received PSL alone. When patients of advanced age receive PSL, even PSL alone, prophylactic TPM-SMX should be administered to prevent the onset of PJP.

Based on the results of this case-control study, we are considering expanding the indications for prophylactic TPMSMX to patients with UC and lymphocyte count $<570 / \mu \mathrm{L}$, even in patients aged $<50$ years old, and to patients aged $\geq 60$ years. We believe that our results will be useful to establish guidelines for prophylactic administration of TPM-SMX.

A limitation of this study was that it was conducted retrospectively at a single institution with limited cases of PJP. A future study that evaluates more patients with PJP at multiple institutions is warranted. Another limitation is that nine cases with PJP were admitted before October 2014, before we started prophylactic TPM-SMX. Consequently, this case-control study does not include cases treated since October 2014 when new immunosuppressive agents became available.

\section{CONCLUSIONS}

Of 4,525 patients with UC, nine (0.2\%) experienced PJP, of whom two died. After starting prophylactic TPM-SMX for UC patients aged $\geq 50$ years who were prescribed three or more immunosuppressive agents, there were no further cases of PJP. The LCI for UC at the onset of PJP was significantly lower than at the initiation of immunosuppressive drug treatment, and the median day of PJP onset was 83 days after the beginning of immunosuppressive treatment. This implies that the onset of PJP occurred at the very time when the symptoms of UC were stabilizing, and the immunosuppressive drugs were being reduced. The risk factors for PJP were senior age, reduced lymphocyte count, and the use of three immunosuppressive drugs. Further, a lymphocyte count of $570.2 / \mu \mathrm{L}$ is a reliable numeral cut-off value to predict the onset of PJP.

Conflicts of interest: None to declare.

Authors' contributions: T.S., M.U., M.S., S.N.: conceived the study. T.S., M.U., T.T., KH, SN: drafted the study. K.K., T.S.: data analysis. K.K. wrote the paper. T.S., M.U., K.H., H.M., H.I., S.N. revised the manuscript. All the authors approved the final version of the manuscript.

\section{REFERENCES}

1. Kovacs JA, Hiemenz JW, Macher AM, et al. Pneumocystis carinii pneumonia: a comparison between patients with the acquired immunodeficiency syndrome and patients with other immunodeficiencies. Ann Intern Med 1984;100:663-671.

2. Limper AH, Offord KP, Smith TF, Martin WJ 2nd. Pneumocystis carinii pneumonia. Differences in lung parasite number and inflammation in patients with and without AIDS. Am Rev Respir Dis 1989;140:12041209. doi:10.1164/ajrccm/140.5.1204

3. Calderón EJ, Gutiérrez-Rivero S, Durand-Joly I, Dei-Cas E. Pneumocystis infection in humans: diagnosis and treatment. Exper Rev Anti Infect Ther 2010;8:683-701. doi:10.1586/eri.10.42

4. Sato T, Inokuma S, Maezawa $\mathrm{R}$, et al. Clinical characteristics of Pneumocystis carinii pneumonia in patients with connective tissue diseases. Mod Rheumatol 2005;15:191-197. doi:10.1007/s10165-0050395-9

5. Iikuni N, Kitahama M, Ohta S, Okamoto H, Kamatani N, Nishinarita M. Evaluation of Pneumocystis pneumonia infection risk factors in patients with connective tissue disease. Mod Rheumatol 2006;16:282-288. doi:10.1007/s10165-006-0502-6

6. Winthrop KL, Park SH, Gul A, et al. Tuberculosis andother opportunistic infections in tofacitinib-treated patients with rheumatoid arthritis. Ann Rheum Dis 2016;75:1133-1138. doi:10.1136/ annrheumdis-2015-207319

7. Long MD, Faraya FA, Okafor PN, Martin C, Sandler RS, Kappelman MD Increased risk of Pneumocystis jirovecii pneumonia among patients with inflammatory bowel disease. Inflamm Bowel Dis 2013;19:10181024. doi:10.1097/MIB.0b013e3182802a9b

8. Cotter TG, Gathaiya N, Catania J, et al. Low risk of Pneumonia from Pneumocystis jirovecii infection in patients with inflammatory bowel disease receiving immune suppression. Clin Gastroenterol Hepatol 2017;15:850-856. doi:10.1016/j.cgh.2016.11.037

9. Tasaka S, Hasegawa N, Kobayashi S, et al. Serum indicators for the diagnosis of Pneumocystis pneumonia. Chest 2007;131:1173-1180. doi:10.1378/chest.06-1467

10. Ferguson ND, Fan E, Camporota L, et al. The Berlin definition of ARDS: an expanded rationale, justification, and supplementary material. Intensive Care Med 2012;38:1573-1582. doi:10.1007/s00134-012-2682-1

11. Kanne JP, Yandow DR, Meyer CA. Pneumocystis jiroveci pneumonia: high-resolution CT findings in patients with and without HIV infection. AJR Am J Roentgenol 2012;198:W555-W561. doi:10.2214/ AJR.11.7329

12. Lichtiger S, Present DH, Kornbluth A, et al. Cyclosporine in severe ulcerative colitis refractory to steroid therapy. N Engl J Med 1994;330:1841-1845. doi:10.1056/NEJM199406303302601

13. Strom B. Sample size considerations for pharmocoepidemiology studies. In: Strom B. (ed.). Pharmacoepidemiology. West Sussex (UK): John Wiley \& Sons; 2000:31-39.

14. Kanda Y. Investigation of the freely available easy-to-use software 'EZR' for medical statistics. Bone Marrow Transplant 2013;48:452-458. doi:10.1038/bmt.2012.244

15. Vananuvat $P$, Suwannalai $P$, Sungkanuparph $S$, Limsuwan $T$, Ngamjanyaporn P, Janwityanujit S. Primary prophylaxis for Pneumocystis jirovecii pneumonia in patients with connective tissue diseases. Semin Arthritis Rheum 2011;41:497-502. doi:10.1016/j. semarthrit.2011.05.004

16. Thomas CF Jr, Limper AH. Pneumocystis pneumonia. N Engl J Med 2004;350:2487-2498. doi:10.1056/NEJMra032588

17. Harigai M, Koike R, Miyasaka N. Pneumocystis pneumonia associated with infliximab in Japan. N Engl J Med 2007;357:1874-1876. doi:10.1056/NEJMc070728

18. Mansharamani NG, Balachandran D, Vernovsky I, Garland R, Koziel H. Peripheral blood CD4+ T-lymphocyte counts during Pneumocystis carinii pneumonia in immunocompromised patients without HIV infection. Chest 2000;118:712-720. doi:10.1378/chest.118.3.712 
19. Okafor PN, Nunes DP, Farraye FA. Pneumocystis jiroveci pneumonia in inflammatory bowel disease: when should prophylaxis be considered? Inflamm Bowel Dis 2013;19:1764-1771. doi:10.1097/ MIB.0b013e318281f562
20. Helweg-Larsen J, Benfield TL, Eugen-Olsen J, Lundgren JD, Lundgren B. Effects of mutation in Pneumocystis carinii dihydropteroate synthase gene on outcome of AIDS-associated P. Carinii Pneumonia. Lancet 1999;354:1347-1351. doi:10.1016/S0140-6736(99)03320-6 